\section{CARIOLOGY; EPIDEMIOLOGY}

\section{Twenty-four month incidence of root} caries among a diverse group of adults

\section{Gilbert G H, Duncan R P, et al. Caries Res 2001; 35: 366-375.}

More than 1/3 of subjects experienced root caries in a large sample biased towards those expected to be at greater risk.

From an initial sample (preferentially weighted towards people who were black, rural dwellers, aged 45 years or older, or poor) of 873 subjects in the Florida Dental Care Study, 723 attended for the 24 month examination; the authors estimated this would cause slight bias towards underestimation of root caries.

At 24 months, 17\% had new root decay only, 14\% had newly filled surfaces and 5\% had both. In those subjects affected, mean DS increment was 2.7 and FS, 1.8. Of 1,117 surfaces with active root caries, 94\% had primary and 6\% recurrent caries. Logistic regression identified four factors significantly associated with new root surface caries or fillings at 24 months: root surface decay, or fillings, or 9-16 teeth present at baseline, or tooth loss during the period. Regular flossing was also associated with significantly less new caries.

\section{DENTAL PUBLIC HEALTH}

\section{Dental diseases and loss of teeth in a group} of Finnish alcoholics: a radiological study

Enberg N, Wolf J, et al. Acta Odontol Scand 2001; 59: 341-347.

The alcoholics had significantly more periodontal bone loss and caries than control subjects.

A group of 85 volunteer Finnish alcoholics (according to DSMIV and ICD-10; mean age 47 yrs) was compared with 53 subjects classified as social drinkers (AUDIT score $=<8 ; 44 \mathrm{yrs}$ ). In addition to being alcohol dependent, $3 / 4$ of the alcoholic group smoked tobacco compared with $1 / 4$ of the controls, 12 alcoholics were unemployed (0 controls) and 10 were on pension or students (4 controls). Panoramic dental radiographs were examined for evidence of disease by two examiners.

The alcoholic group had significantly fewer teeth (mean 22 v. 27), more teeth with caries, calculus or infrabony pockets, and similar numbers of teeth with periapical lesions or past endodontic treatment. A significantly higher number of nonsmoking alcoholics had destructive periodontal disease than did controls. The authors consider the poorer dental condition of the alcoholics to be a consequence of their social condition.

\section{BEHAVIOURAL SCIENCES}

\section{Psychological disorders and dental anxiety in a young adult population}

Locker D, Poulton R, et al. Community Dent Oral Epidemiol 2001; 29: 456-463.

In a New Zealand birth cohort study group, those with high dental anxiety also had high rates of other psychological problems.

In 1972-3, a cohort of 1,037 children born in a Dunedin hospital was followed longitudinally with medical, dental, psychological and sociological data collected at intervals. At ages 18 and 26 years, dental anxiety and psychological data were examined for 805 subjects.

The mean Dental Anxiety Score (DAS) at 18 years was 8.5, with $12.5 \%$ considered anxious, having scores of $13+$. Of these anxious subjects, 55\% had one or more psychological disorders, compared with $42 \%$ of other subjects $(\mathrm{P}<0.05)$. The commonest disorders were: major depression, alcohol dependence and social phobia.

DAS scores did not predict dental anxiety at 26 years, but those with psychological disorders at age 18 were more likely to be anxious at age 26 . The authors suggest that dental anxiety is a distinct state, because those with mood disorders did not show it more frequently.

\section{DENTAL OCCLUSION}

The relationship between non-working-side occlusal contacts and mandibular position

Ogawa T, Ogimoto T, et al. J Oral Rehabil 2001; 28: 976-981.

In a group of 89 normocclusive adults (ages 20-29 yrs), contacts varied with mandibular position.

This study examined well defined mandibular positions in relation to lateral excursive contacts on the non-working side of the arch. From the position of maximum intercuspation, lines were marked on the maxillary central incisor to give lateral excursions at 0.5, 1, 2 and $3 \mathrm{~mm}$.

There were fewer than 3\% non-working contacts on all teeth except 1 st and 2 nd molars. These also decreased markedly and significantly with increasing mandibular excursion, from about $20 \%$ and $30 \%$ respectively at $0.5 \mathrm{~mm}$. At the four lateral excursive positions, total contacts decreased from $40 \%$ to around 10\%. Contacts were commoner with group function than with canine protection (at $0.5 \mathrm{~mm}, 48 \% \mathrm{v} .11 \%$ ).

The authors comment that it does not seem that non-working contact is harmful to teeth, but it may be important in masticatory function. They suggest that the $0.5 \mathrm{~mm}$ position should be used to assess function, and the $3 \mathrm{~mm}$ position for parafunction. 